\title{
Are Menstrual and Nonmenstrual Migraine Attacks Different?
}

\author{
Kjersti Grøtta Vetvik • Michael Bjørn Russell
}

Published online: 17 May 2011

(C) The Author(s) 2011. This article is published with open access at Springerlink.com

\begin{abstract}
Migraine is the second most common headache condition next to tension-type headache. Up to one fourth of all women have migraine, and $20 \%$ of them experience migraine without aura attack in at least two thirds of their menstrual cycles. The current literature is analyzed in response to the question of whether menstrual and nonmenstrual migraine attacks are different. The different studies provide conflicting results, so it is not possible to answer the question firmly. Future studies should be based on the general population. Collection of both prospective and retrospective data is warranted, and headache diagnosis base on interviews by physicians with interest in headache are more precise than lay interviews or questionnaires.
\end{abstract}

Keywords Menstrual migraine - Symptomatology

Disability and treatment . Perimenstrual headache .

Nonmenstrual migraine $\cdot$ Attacks $\cdot$ Rizatriptan $\cdot$ Almotriptan

\section{Introduction}

The appendix of the second edition of the International Classification of Headache Disorders (ICHD-2) from 2004 describes two types of migraine without aura (MO) related to menstruation: pure menstrual migraine (PMM) and

K. G. Vetvik • M. B. Russell ( $ه)$

Head and Neck Research Group, Research Centre,

Akershus University Hospital,

1478 Lørenskog, Oslo, Norway

e-mail: m.b.russell@medisin.uio.no

K. G. Vetvik • M. B. Russell

Institute of Clinical medicine, Campus Akershus University

Hospital, University of Oslo,

1478 Lørenskog, Oslo, Norway menstrually related migraine (MRM) [1]. Those with PMM have MO attacks exclusively in relation to at least two thirds of their menstrual periods, while those with MRM additionally experience MO attacks outside the menstrual period. Before the ICHD-2 classification, researchers had no specific guidelines for the definition of menstrual migraine. The first classification of the International Headache Society from 1988 did not classify menstrual migraine as a specific type of headache, but had a comment in relation to MO: "Migraine without aura may occur almost exclusively at a particular time of the menstrual cycleso-called menstrual migraine. Generally accepted criteria for this entity are not available. It seems reasonable to demand that $90 \%$ of attacks should occur between 2 days before menses and the last day of menses, but further epidemiological knowledge is needed" [2]. The latter definition encompasses only a few of those with MRM. A recent epidemiological survey suggests that $21 \%$ of women with migraine have menstrual migraine, and about two thirds have MRM [3•]. These circumstances make the literature on menstrual migraine heterogenous, which one should bear in mind in the current analysis of symptomatology of menstrual and nonmenstrual migraine attacks. To complicate matters, what about migraine with aura (MA)? About one third of all migraineurs have MA [4]. An epidemiological survey implies that headache in attacks of MO may be slightly more severe than headache in attacks of MA [5], but it seems that MA is not associated with menstruation on the same scale as MO. In fact, MA may be unrelated to menstruation [6].

The most precise information on migraine attacks is ascertained from prospective recording (ie, headache diaries with detailed information about pain characteristics, accompanying symptoms, duration, and treatment). Such data may not be generalizable because keeping a headache diary for a longer period is not applicable for a large population 
due to low compliance. In contrast, retrospective data from large population-based studies may be generalizable, but the detailed information about migraine attacks is subject to memory bias. Thus, a combination of prospective and retrospective data is likely to give the most precise description of the migraine attacks.

This review analyzes the literature to enlighten whether menstrual and nonmenstrual migraine attacks are different, given the methodological challenges mentioned above.

\section{Menstrual Versus Nonmenstrual Migraine Attacks}

\section{Attack Frequency}

A Danish epidemiological survey based on 18- to 65-year-old people from the general population, including a direct clinical interview and a physical and neurological examination by a physician, found that less than $10 \%$ of women with MO had more than one $\mathrm{MO}$ attack per month [5].

Thus, the vast majority (ie, $\geq 90 \%$ ) of all women with migraine have 1 or fewer MO attacks per month. Some of those with PMM will have $1 \mathrm{MO}$ attack per month, while most patients with MRM have more than $1 \mathrm{MO}$ attack per month. Thus, the MO attack frequency is much higher in most women with PMM or MRM than in those with nonmenstrual MO. Although this does not address whether menstrual and nonmenstrual MO attacks are different, it indicates that those with menstrual MO are likely to have a higher disease burden than most women with nonmenstrual MO.

\section{Attack Severity}

\section{Pain Characteristics}

Table 1 shows attack characteristics of menstrual migraine in relation to nonmenstrual migraine. Two studies from the general population suggest that the pain intensity is higher in menstrual than nonmenstrual MO [7, 8]. Studies on clinic populations provide conflicting results; four studies are in support of the findings in the general population, while four studies suggest similar pain intensity and one study missed information on pain intensity $[9-15,16 \bullet 17 \bullet]$.

\section{Associated Symptoms}

The study based on prospective filled-in diaries from the general population suggests no significant difference in associated symptoms in attacks of menstrual and nonmenstrual migraine [7]. This is supported by three studies based on clinic populations $[10,15,16 \cdot]$. However, two clinic studies based on prospective filled-in diaries suggest that menstrual migraine attacks are accompanied by more nausea and vomiting than nonmenstrual attacks $[11,14]$.

\section{Duration}

The study based on prospective filled-in diaries from the general population suggests that attacks of menstrual and nonmenstrual migraine have similar duration [7]. In contrast, all the other studies suggest that attacks of menstrual migraine are longer than attacks of nonmenstrual migraine, including data based on prospective filled-in diaries $[8,10,16 \bullet 17 \bullet]$.

\section{Disability}

The study based on prospective filled-in diaries from the general population and one clinic study suggest that attacks of menstrual and nonmenstrual migraine have similar disability $[7,15]$. In contrast, all the other studies suggest that attacks of menstrual migraine are more disabling than attacks of nonmenstrual migraine, including data based on prospective filled-in diaries $[8,10,12,13,16 \bullet 17 \bullet]$.

\section{Treatment}

Two clinical trials on rizatriptan and almotriptan suggest equal efficacy on pain relief $2 \mathrm{~h}$ post-treatment of menstrual and nonmenstrual migraine attacks $[9,15]$. Other studies suggest that attacks of menstrual migraine are more treatment resistant than attacks of nonmenstrual migraine $[8,10,16 \cdot]$ or that menstrual migraine attacks are more likely to relapse $[17 \bullet]$.

\section{Conclusions}

The question whether menstrual and nonmenstrual migraine attacks are different unfortunately cannot be answered unambiguously because previous studies provide conflicting results. This is mainly due to different patient populations, unequal definitions of menstrual migraine, and different methods employed.

Future studies should focus on the general population to provide generalizable data, in contrast to possibly skewed data from selected clinic populations. Prospective recordings should be employed, combined with retrospective data on the headache history. To provide precise headache diagnoses, interviews by a trained physician or neurologist with special interest in headache is the gold standard. Such a study is likely to more firmly answer whether menstrual and nonmenstrual migraine attacks are different. 


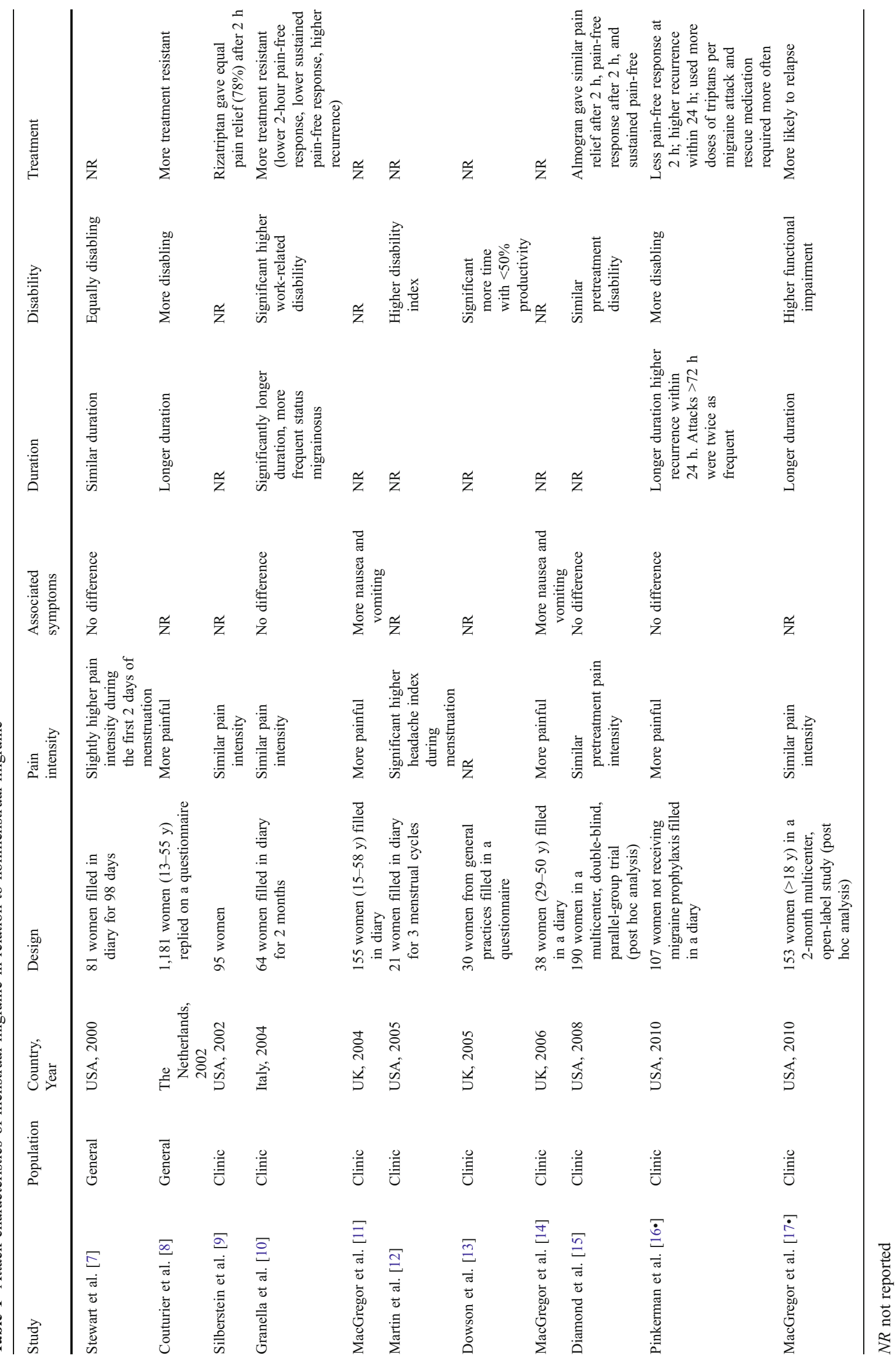


Disclosures Dr. K. G. Vetvik: none. Dr. Michael B. Russell has served as a board member and consultant for Allergan.

Open Access This article is distributed under the terms of the Creative Commons Attribution Noncommercial License which permits any noncommercial use, distribution, and reproduction in any medium, provided the original author(s) and source are credited.

\section{References}

Papers of particular interest, published recently, have been highlighted as:

- Of importance

1. Headache Classification Subcommittee of the International Headache Society. The international classification of headache disorders: 2nd edition. Cephalalgia. 2004;24 Suppl 1:9-160.

2. Classification and diagnostic criteria for headache disorders, cranial neuralgias and facial pain. Headache Classification Committee of the International Headache Society. Cephalalgia. 1988;8(Suppl 7):1-96.

3. - Vetvik KG, MacGregor EA, Lundqvist C, Russell MB. Selfreported menstrual migraine in the general population. J Headache Pain. 2010;11:87-92. This paper provides prevalence data on menstrual migraine based on a population-based sample of 15,000 women.

4. Russell MB, Rasmussen BK, Thorvaldsen P, Olesen J. Prevalence and sex-ratio of the subtypes of migraine. Int $\mathrm{J}$ Epidemiol. 1995;24:612-8

5. Rasmussen BK, Olesen J. Migraine with aura and migraine without aura: an epidemiological study. Cephalalgia. 1992;12:221-8.

6. Russell MB, Rasmussen BK, Fenger K, Olesen J. Migraine without aura and migraine with aura are distinct clinical entities: a study of four hundred and eighty-four male and female migraineurs from the general population. Cephalalgia. 1996;16:239-45.

7. Stewart WF, Lipton RB, Chee E, Sawyer J, Silberstein SD. Menstrual cycle and headache in a population sample of migraineurs. Neurology. 2000;55:1517-23.
8. Couturier EG, Bomhof MA, Neven AK, van Duijn NP. Menstrual migraine in a representative Dutch population sample: prevalence, disability and treatment. Cephalalgia. 2003;23:302-8.

9. Silberstein SD, Massiou H, McCarroll KA, Lines CR. Further evaluation of rizatriptan in menstrual migraine: retrospective analysis of long-term data. Headache. 2002;42:917-23.

10. Granella F, Sances G, Allais G, Nappi RE, Tirelli A, Benedetto C, et al. Characteristics of menstrual and nonmenstrual attacks in women with menstrually related migraine referred to headache centres. Cephalalgia. 2004;24:707-16.

11. MacGregor EA, Hackshaw A. Prevalence of migraine on each day of the natural menstrual cycle. Neurology. 2004;63:351-3.

12. Martin VT, Wernke S, Mandell K, Ramadan N, Kao L, Bean J, et al. Defining the relationship between ovarian hormones and migraine headache. Headache. 2005;45:1190-201.

13. Dowson AJ, Kilminster SG, Salt R, Clark M, Bundy MJ. Disability associated with headaches occurring inside and outside the menstrual period in those with migraine: a general practice study. Headache. 2005;45:274-82.

14. MacGregor EA, Frith A, Ellis J, Aspinall L, Hackshaw A. Incidence of migraine relative to menstrual cycle phases of rising and falling estrogen. Neurology. 2006;67:2154-8.

15. Diamond ML, Cady RK, Mao L, Biondi DM, Finlayson G, Greenberg SJ, et al. Characteristics of migraine attacks and responses to almotriptan treatment: a comparison of menstrually related and nonmenstrually related migraines. Headache. 2008;48:248-58.

16. - Pinkerman B, Holroyd K. Menstrual and nonmenstrual migraines differ in women with menstrually-related migraine. Cephalalgia. 2010;30:1187-94. This was a prospective study based on a diary-confirmed ICHD-2 diagnosis of menstrual migraine in women with frequent and severe migraine not taking migraine prophylaxis.

17. - MacGregor EA, Victor TW, Hu X, Xiang Q, Puenpatom RA, Chen $\mathrm{W}$, et al. Characteristics of menstrual vs nonmenstrual migraine: a post hoc, within-woman analysis of the usual-care phase of a nonrandomized menstrual migraine clinical trial. Headache. 2010;50:528-38. This study recruited women from primary care to provide a within-woman analysis of menstrual and nonmenstrual attacks based on prospectively filled-in diaries. 